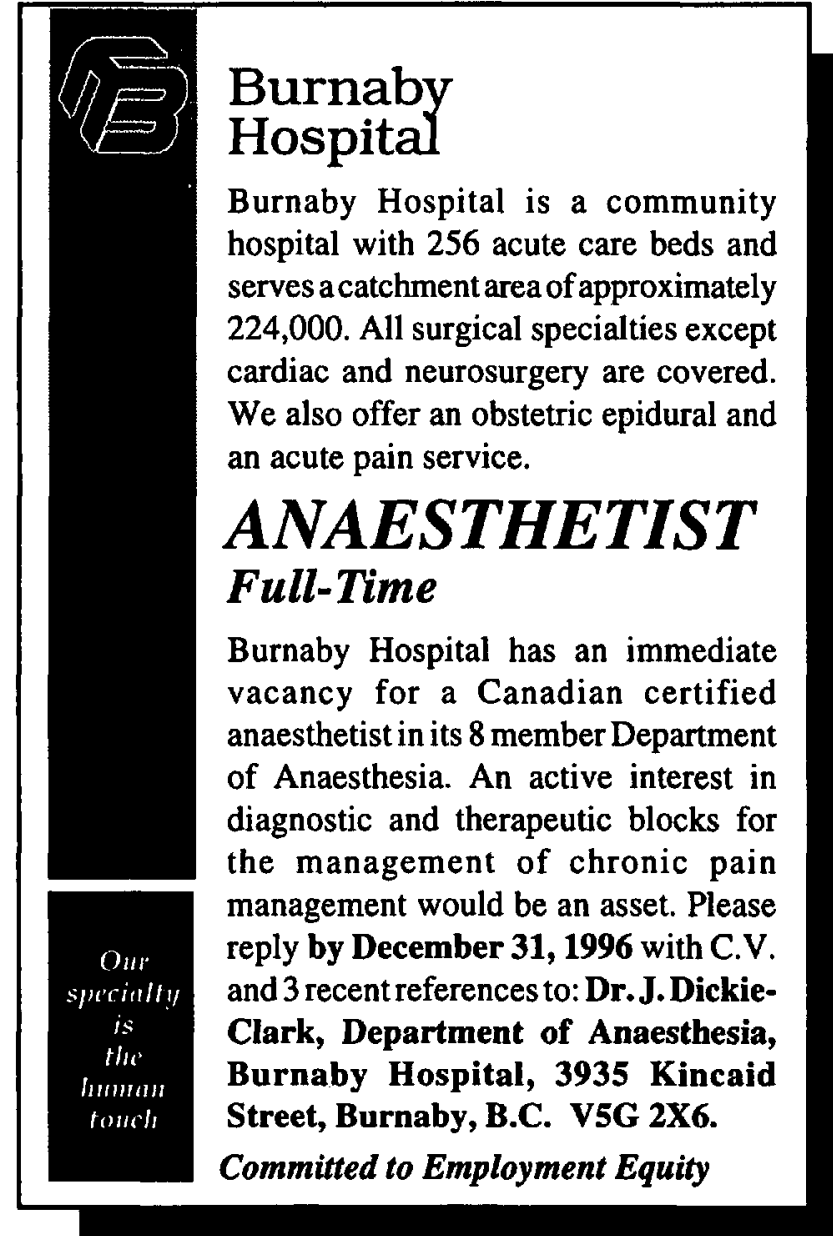

\section{YOUR ANNOUNCEMENT COULD APPEAR IN THIS SPOT!}

As a regular reader of the Canadian Journal of Anaesthesia you are aware of the high quality of our publication. High quality bas translated into a large readership. At present, the CJA is sent to over 5,000 individuals and institutions across Canada, the United States and overseas, with a large hidden readership at major hospitals and medical libraries worldwide.

This is your monthly target audience for announcements covering:

Positions Available

Positions Wanted

Meetings

Postgraduate Courses

Other Events

Call our sales representatives for further details and to book space in the next available issue!

Canadian Journal of Anaesthesia

c/o Keith Health Care Communications

1382 Hurontario Street

Mississauga, ON

Canada

Phone: (905) 278-6700 Fax: (905) 278-4850
Winterlude '97Anaesthesla Symposium - University of Ottawa

Monitoring, Equipment and Technique:

Foundations for Clinical Acumen

Chateau Laurier Hotel

Saturday, February 8,1997

\section{Moming Session:}

Intraoperattve Braln Monitoring - Dr. R. Rodriguez - Ottawa

Body Temperature - Dr. L. Gamett - Ottawa

The Pattent Intolerant of One-Lung Ventilation - Dr. P. Singer - Toronto

Cardlac Monitoring 1997: The Role of TEE - Dr. M. Hynes - Ottawa

New Monitors: The Cost vs. Quality Dilemma - Dr. C. Eagle - Calgary

Aftemoon Small-Group Sessions:

Choose any 3 of these $S$ Toplcs:

Top/c A: Upper Alrway Management - Dr. E. Crosby

Topic B: Lung Isolation Techniques - Dr. P. Slinger

Topic C: Intro to Transesoph. Echocardlography - Dr. I. Thomson

- Winnipes

Topic D: Troubleshooting Gas Machines and Monitors - Dr. R. Elliott

Topic E: Anaesthesla On-Line: Intemet Basics - Dr. P. Duffy - Dr. J. Oyston - Orllila

\section{MEW! Special Resident Session:}

Frtday, February 7, 1997 - 14.00-16:30

The Oral Exam: Survival Tips and Techniques

$$
\text { - Dr. C. Eagle and Dr. J. McKenna }
$$

\begin{tabular}{|c|c|}
\hline $\begin{array}{r}\text { For inrormation } \\
\text { Conract: } \\
\text { (613) } 761-4940\end{array}$ & $\begin{array}{l}\text { Ms. Lynne McHardy, Secretary } \\
\text { Winterlude ' } 97 \text { Symposium, Dept. Anaesthesia } \\
\text { Ottawa Civic Hospilal - B3 } \\
\text { 1053 Carling Ave., Ottawa ON K1Y 4E9 }\end{array}$ \\
\hline
\end{tabular}

Winterlude '97Anaesthesla Symposium - University of Ottawa

\section{McGill University}

Continuing Medical Education

\section{9th Annual Review Course in Anaesthesia Montreal, May 24-27 1997}

A comprehensive lecture and workshop program with themes including:

Airway/Thoracic, Cardiac, Neuroscience, Paediatrics, Pharmacology, Obstetrics, Regional Anaesthesia,

Practice Issues and a special review for Respiratory Technologists

Presented by an international faculty of leading authorities

Fees before March 28, 1997

Physicians: \$600Cdn / \$450US Residents: \$500Cdn / \$375US

Fees after March 28, 1997 Physicians: \$650Cdn / \$48SUS Residents: $\$ 550 \mathrm{Cdn} / \$ 410 \mathrm{US}$

\section{ALL On-Site registrations $\$ 700$ Cdn}

For program and information contact:

Joanne Kaplo

McGill University, Continuing Medical Education 3655 Drummond Street, Suite 529 Montreal, QC H3G 1 Y6

Tel: (514) 398-3340 Fax: (514) 398-3595 